\title{
Epidemiology of Fracture in Adults from Ontario, Canada, with Chronic Kidney Disease: An Examination of Fracture Burden Using Administrative Health Data
}

\author{
Kyla L. Naylor, Amit X. Garg, S. Joseph Kim and Gregory A. Knoll
}

\begin{abstract}
Fractures are associated with morbidity and mortality. Individuals with chronic kidney disease (CKD) experience bone mineral metabolism changes, which increases fracture risk. Researchers have quantified the epidemiology of fractures in adults with CKD using administrative health databases from Ontario, Canada, held at the Institute for Clinical Evaluative Sciences. Results demonstrated that many individuals with non-transplant CKD sustain fractures, with the risk increasing as kidney function declines. However, fracture risk in kidney transplant recipients was lower than previously described, which suggests recipients may not be a high-risk fracture group. There is a need to test fracture prevention interventions in the CKD population.
\end{abstract}

\section{The Issue}

The number of chronic kidney disease (CKD) patients in Canada is increasing, with over three million adults living with CKD (Arora et al. 2013; CIHI 2013). There is a concern about poor outcomes associated with CKD, one of which is fractures. Several studies assessing the epidemiology of fracture in CKD have been conducted using administrative databases in the United States. However, these findings may not accurately reflect the Canadian experience, as fracture rates have been shown to vary as much as 15-fold across countries (Kanis et al. 2002). Access to large healthcare databases in Ontario, Canada, provides an opportunity to conduct a comprehensive examination of the epidemiology of fracture in the CKD population.

Multiple factors likely contribute to an increased fracture risk in the CKD population. As kidney function declines, CKD-related mineral and bone disorders may develop (i.e., changes in calcium, phosphorous, vitamin $\mathrm{D}$ or parathyroid hormone metabolism), and this can lead to an increased fracture risk (Kidney Disease Improving Global Outcomes [KDIGO] 2009). Other factors may also increase fracture risk, such as muscle wasting (West et al. 2012) and steroid administration to kidney transplant recipients (Canalis et al. 2007). Despite this increased fracture risk, there remain many unanswered questions about interventions that will safely prevent fractures in the CKD population (KDIGO 2009; Palmer et al. 2009). Moreover, many of the guideline statements for the treatment and evaluation of bone disease in CKD patients are weak or ungraded (KDIGO 2009). An improved understanding of the epidemiology of fracture in the CKD population informs the conduct of well-designed clinical trials and prospective studies.

This report highlights several recent studies conducted at the Institute for Clinical Evaluative Sciences (ICES) by the provincial Kidney, Dialysis and Transplantation team aimed to clarify fracture incidence, improve clinical guidelines, advance prognostication and guide informed consent to help decrease the fracture burden in the CKD population.

\section{Key Findings \\ Fracture incidence in the non-transplant chronic kidney disease population}

Knowing an individual's fracture risk according to kidney function, age and sex is useful to guide sample-size estimates for future fracture prevention trials and for risk stratification. ICES databases were used to determine the three-year cumulative incidence of fracture (defined as a composite of hip, pelvis, forearm or proximal humerus fractures) stratified by age (40-65 and $>65$ years), sex and kidney function (as measured by an estimated glomerular filtration rate $[\mathrm{eGFR}]$ of $>60 \mathrm{~mL} / \mathrm{min} / 1.73 \mathrm{~m}^{2}$, $45-59,30-44,15-29$ and $<15$ or chronic dialysis) at cohort entry (2002-2009) (Naylor et al. 2014). As kidney function declined, 
fracture risk increased in a graded manner. For example, in women $>65$ years, the three-year cumulative incidence of fracture across the five eGFR categories was $4.3 \%, 5.8 \%, 6.5 \%, 7.8 \%$ and $9.6 \%$ over the study follow-up (Figure 1). In other words, 1 in 10 women aged $>65$ years with an eGFR of $<15 \mathrm{~mL} / \mathrm{min} / 1.73 \mathrm{~m}^{2}$ will experience a fracture over a three-year follow-up. Similar results were found in men $(1.6 \%, 2.0 \%, 2.7 \%, 3.8 \%$ and $5.0 \%)$ (Figure 2). Even individuals with moderate declines in kidney function had a significantly higher fracture risk compared to those with normal kidney function. For example, women aged $>65$ years with an eGFR of $45-59 \mathrm{~mL} / \mathrm{min} / 1.73 \mathrm{~m}^{2}$ had a 1.4 -times higher fracture risk compared to women with an eGFR of $>60 \mathrm{~mL} / \mathrm{min} / 1.73 \mathrm{~m}^{2}$.

\section{FIGURE 1.}

Three-year cumulative incidence of fracture in women displayed by kidney function. From: Naylor et al. 2014; reprinted with permission from Elsevier

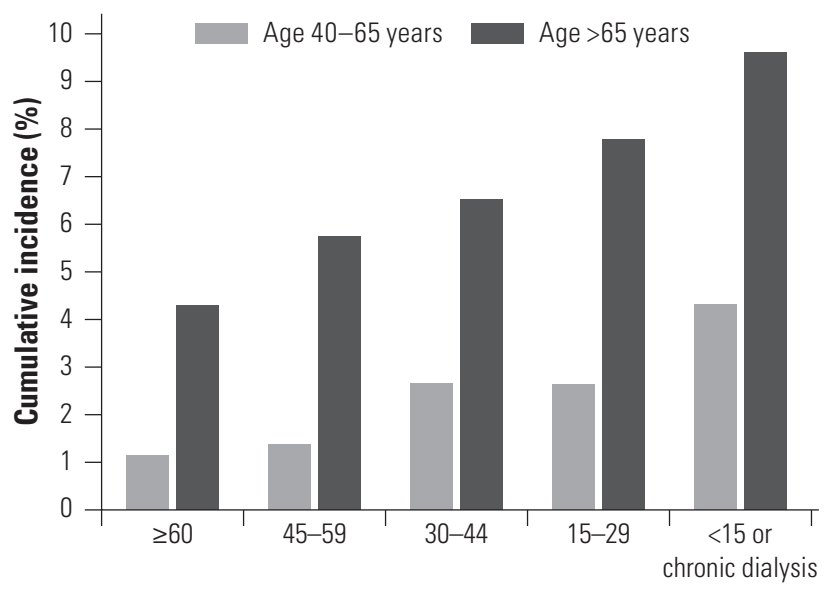

Estimated glomerular filtration rate $\left(\mathrm{mL} / \mathrm{min} / 1.73 \mathrm{~m}^{2}\right)$

FIGURE 2.

Three-year cumulative incidence of fracture in men displayed by kidney function. From: Naylor et al. 2014; reprinted with permission from Elsevier

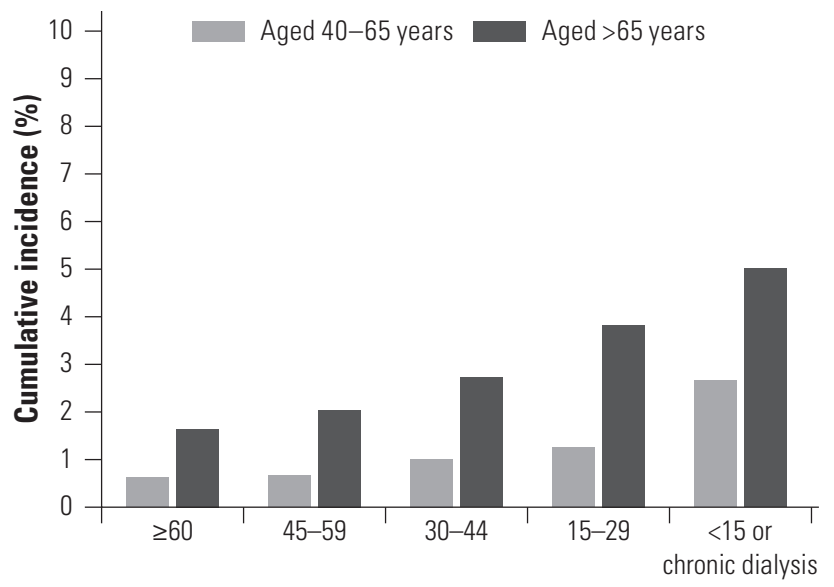

Estimated glomerular filtration rate $\left(\mathrm{mL} / \mathrm{min} / 1.73 \mathrm{~m}^{2}\right)$
Falls increase the risk of fracture, and preventing falls is an essential strategy in fracture prevention (Cumming and Klineberg 1994; Papaioannou et al. 2010). ICES databases were used to assess the three-year cumulative incidence of falls resulting in a hospital encounter. Similar to fractures, as kidney function declined, fall risk increased in a graded manner. For example, the three-year cumulative incidence of falls in women aged $>65$ years across the five eGFR categories was 3.8\%, 5.9\%, 7.6\%, 9.1\% and 13.1\% (Naylor et al. 2014).

\section{Fracture incidence in kidney transplant recipients}

To help quantify fracture risk (defined as a composite of hip, forearm or proximal humerus fractures), kidney transplant recipients who received a transplant in Ontario between 1994 and 2009 were compared with two groups at high risk for fracture (non-dialysis CKD population and a healthy segment of the general population with previous fractures) and one low-risk group (healthy segment of the general population with no previous fracture) matched for age, sex and cohort entry date (Naylor et al. 2016a). Kidney transplant recipients had a significantly higher three-year cumulative incidence of fracture $(1.6 \%)$ compared with the non-dialysis CKD population $(1.1 \%)$ and the general population with no previous fracture $(0.5 \%)$. However, when compared with the general population with a previous fracture $(2.3 \%)$, recipients had a significantly lower fracture risk. Kidney transplant recipients were not considered a high-risk group for fracture as only $1.7 \%$ of them sustained a hip fracture over 10 years (clinical practice guidelines define high-risk as a 10-year risk of hip fracture of $\geq 3 \%$ ) (Grossman et al. 2010; National Osteoporosis Foundation 2010).

Similar to the non-transplant CKD population, falls involving a hospital encounter among kidney transplant recipients were common, with a three-year cumulative incidence of $7.9 \%$ (Naylor et al. 2016a). Female recipients aged $\geq 50$ years had a particularly high fall risk, with 1 in 10 (11.1\%) sustaining a fall in the three years after transplant.

\section{Bone mineral density testing in kidney transplant recipients}

In the general population, several measures have been recommended by Osteoporosis Canada to assess bone health. For example, bone mineral density (BMD) tests are widely used to risk-stratify patients and help guide treatment decisions (Papaioannou et al. 2010). However, given the complex pathophysiology of bone disease among kidney transplant recipients, the utility of BMD in this population is controversial (KDIGO 2009). Despite limited evidence supporting the widespread use of BMD among recipients, more than half (58\%) of Ontario kidney transplant recipients who received a transplant between 1994 and 2009 had at least one BMD test in the three years after transplant, with approximately $30 \%$ having more than two 
tests (Naylor et al. 2016b). In total, these tests cost more than $\$ 600,000$ (in 2014 Canadian-equivalent dollars). The number of recipients who were administered at least one BMD test significantly increased over time (recipients who transplanted in $1994,20.9 \%$; in $2009,66.4 \%$ ). Recipients were significantly more likely to receive at least one BMD test $(58.0 \%)$ compared with the general population with no previous fracture (8.5\%) and the general population with a previous fracture (13.8\%), matching on age, sex and cohort entry date. There was significant variation across the six Ontario transplant centres in the proportion that performed at least one BMD test in the three years post-transplant (from $15.6 \%$ to $92.1 \%$ ), and this variability persisted after controlling for factors likely to influence a clinician's decision to perform a test (e.g., age).

\section{Implications}

Many individuals with CKD will fracture and fall. Although fracture risk in kidney transplant recipients was lower than expected, recipients still had a significantly higher fracture risk compared with the healthy general population with no previous fracture. As the Canadian CKD and kidney transplant populations continue to grow with improved survival rates, fractures and their associated morbidity will become an increasing concern. A shift in bone-disease research and resources towards this understudied population could be helpful.

These findings have implications for family physicians. Individuals with mild to moderate declines in kidney function are often primarily managed by family physicians, and kidney transplant recipients are often managed in collaboration with nephrologists. Therefore, family physicians can play an integral role in fracture management in the $\mathrm{CKD}$ population.

\section{... fractures and their associated morbidity will become an increasing concern.}

While serious falls are common in the CKD population, KDIGO (2009) guidelines for CKD-related mineral and bone disorders only minimally discuss them. However, Osteoporosis Canada guidelines provide an in-depth discussion of falls in the general population as they state their management is an integral part of fracture prevention (Papaioannou et al. 2010). Future CKD guidelines should incorporate a section on falls. Moreover, the efficacy of fall prevention programs in this unique patient population needs to be determined.

Given the burden of fractures in the CKD population, studies that test promising interventions to prevent fracture are needed. According to the KDIGO guidelines, there is insufficient information to guide treatment for fracture prevention one year post-transplant, which may be attributable to clinical trials having inadequate statistical power (KDIGO
2009; Palmer et al. 2009). Moreover, the safety and efficacy of fracture prevention therapies in individuals with an eGFR of $<30 \mathrm{~mL} / \mathrm{min} / 1.73 \mathrm{~m}^{2}$ is uncertain (KDIGO 2013; Lin 1996). Therefore, adequately powered clinical trials are required. Based on three-year cumulative incidence estimates obtained from these ICES studies, thousands of CKD patients would need to be enrolled in clinical trials to ensure sufficient power. This can only be accomplished through a collaboration of multiple centres caring for CKD patients. Prospective studies are also required to determine the utility, timing and costeffectiveness of BMD testing in kidney transplant recipients.

In summary, using administrative health databases from ICES, the epidemiology of fractures in Ontario CKD patients was quantified. Given the growing burden of CKD, strategies to prevent fractures and falls are needed to improve outcomes in this patient population. $\mathrm{HQ}$

\section{References}

Arora, P., P. Vasa, D. Brenner, K. Iglar, P. McFarlane, H. Morrison et al. 2013. "Prevalence Estimates of Chronic Kidney Disease in Canada: Results of a Nationally Representative Survey." CMAJ 185(9): E417-23.

Canadian Institute for Health Information (CIHI). 2013. Canadian Organ Replacement Register Annual Report: Treatment of End-Stage Organ Failure in Canada, 2003 to 2012. Ottawa, ON: Author. Retrieved March 16, 2016. <https://secure.cihi.ca/free_products/2014_CORR_ Annual_Report_EN.pdf>.

Canalis, E., G. Mazziotti, A. Giustina and J.P. Bilezikian. 2007. "Glucocorticoid-Induced Osteoporosis: Pathophysiology and Therapy.” Osteoporosis International 18(10): 1319-28.

Cumming, R.G. and R.J. Klineberg. 1994. "Fall Frequency and Characteristics and the Risk of Hip Fractures." Journal of the American Geriatrics Society 42(7): 774-78.

Grossman, J.M., R. Gordon, V.K. Ranganath, C. Deal, L. Caplan, W. Chen et al. 2010. "American College of Rheumatology 2010 Rrecommendations for the Prevention and Treatment of Glucocorticoid-Induced Osteoporosis." Arthritis Care and Research (Hoboken) 62(11): 1515-26.

Kanis, J.A., O. Johnell, C. De Laet, B. Jonsson, A. Oden and A.K. Ogelsby. 2002. "International Variations in Hip Fracture Probabilities: Implications for Risk Assessment." Journal of Bone and Mineral Research 17(7): 1237-44.

Kidney Disease: Improving Global Outcomes (KDIGO) CKD-MBD Work Group. 2009. "KDIGO Clinical Practice Guidelines for the Diagnosis, Evaluation, Prevention, and Treatment of Chronic Kidney Disease-Mineral and Bone Disorder (CKD-MBD)." Kidney international 76(Suppl 113): S1-130.

Kidney Disease: Improving Global Outcomes (KDIGO) Work Group. 2013. "KDIGO 2012 Clinical Practice Guideline for the Evaluation and Management of Chronic Kidney Disease." Kidney International Supplements 3(1): 1-150.

Lin, J.H. 1996. "Bisphosphonates: A Review of Their Pharmacokinetic Properties.” Bone 18(2): 75-85. 
National Osteoporosis Foundation. 2010. Clinician's Guide to Prevention and Treatment of Osteoporosis. Washington, DC: Author.

Naylor, K.L., E. McArthur, W.D. Leslie, L.A. Fraser, S.A. Jamal, S.M. Cadarette et al. 2014. "The Three-Year Incidence of Fracture in Chronic Kidney Disease.” Kidney International 86(4): 810-18.

Naylor, K.L., S.A. Jamal, G. Zou, E. McArthur, N.N. Lam, W.D. Leslie et al. 2016a. "Fracture Incidence in Adult Kidney Transplant Recipients." Transplantation 100(1): 167-75.

Naylor, K.L., G.Y. Zou, W.D. Leslie, E. McArthur, N.N. Lam, G.A. Knoll et al. 2016b. "Frequency of Bone Mineral Density Testing in Adult Kidney Transplant Recipients from Ontario, Canada: A Population-Based Cohort Study." Canadian Journal of Kidney Health and Disease 3: 2.

Palmer, S.C., D.O. McGregor and G.F. Strippoli. 2009. "Interventions for Preventing Bone Disease in Kidney Transplant Recipients." Cochrane Database of Systematic Reviews (3): CD005015.

Papaioannou, A., S. Morin, A.M. Cheung, S. Atkinson, J.P. Brown, S. Feldman et al. 2010. Clinical Practice Guidelines for the Diagnosis and Management of Osteoporosis in Canada: Background and Technical Report. Toronto, ON: Osteoporosis Canada. Retrieved March 16, 2016. <http://www.osteoporosis.ca/multimedia/pdf/Osteoporosis_ Guidelines_2010_Background_And_Technical_Report.pdf>.

West, S.L., S.A. Jamal and C.E. Lok. 2012. "Tests of Neuromuscular Function are Associated with Fractures in Patients with Chronic Kidney Disease.” Nephrology, Dialysis, Transplantation 27(6): 2384-88.

\section{About the Authors}

Kyla L. Naylor, PhD, is a postdoctoral research fellow at the Institute for Clinical Evaluative Sciences and at the University of Toronto's Institute of Health Policy, Management and Evaluation. She has received a Canadian Institutes of Health Research Fellowship and a Canadian National Transplant Research Program Astellas Training Award. She may be contacted at kyla.naylor@ices.on.ca.

Amit X. Garg, MD, PhD, FRCPC, is a nephrologist at London Health Sciences Centre, a professor of medicine, epidemiology and biostatistics at Western University, lead of the ICES KDT research program, and an ICES scientist.

S. Joseph Kim, MD, PhD, MHS, FRCPC, is a nephrologist at Toronto General Hospital, an assistant professor at the Institute of Health Policy, Management and Evaluation at the University of Toronto, associate lead of the ICES KDT program, and an ICES scientist.

Gregory A. Knoll, MD, MSc, FRCPC, is a nephrologist and medical director of renal transplantation at The Ottawa Hospital, professor of medicine at the University of Ottawa, scientist at The Ottawa Hospital, the chair in Clinical Transplantation at the University of Ottawa and vice chair of research in the department of medicine at The Ottawa Hospital.

\footnotetext{
.
}

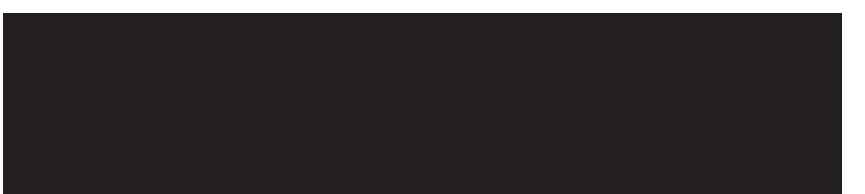

\section{Breakfast with} the Chiefs 